\title{
Gross Examination of the Placenta and Its Importance in Evaluating an Unexplained Intrauterine Fetal Demise
}

\author{
Sunil Jaiman ${ }^{1}$
}

Received: 19 July 2015/Accepted: 12 October 2015/Published online: 30 October 2015

(C) Society of Fetal Medicine 2015

\begin{abstract}
Placenta, a fascinating multifunctional organ, of fetal origin, plays a pivotal conciliatory role during pregnancy by being intimately connected to the mother and her baby. The importance of placenta is irrefutable, being an exceptional (and easily-accessible) source of information which echoes the intrauterine environment. Placental examination can shed light upon factors pertaining to the current pregnancy and its outcome, guide postpartum management, provide insight into clinical problems (such as seizures, pulmonary hypertension, renal failure) that become evident hours or days after delivery, be predictive of future pregnancies, and even play a role in medico-legal situations. The rhetoric that since most babies are normal so are the placentas, therefore an examination of all placentas may not be warranted, has fallen in disfavor and currently placental examination even for 'routine' deliveries is being advocated. Until such time, this becomes an established practice examination of the placenta should certainly be performed in any maternal or fetal complications and stillbirths. The placenta can provide exceedinglyuseful information relating to perinatal morbidity and mortality. Furthermore, it has been shown repeatedly that a placental examination is needed to understand the causes of perinatal deaths. In order to derive clinically-useful information from the placenta, meticulous macroscopic examination is the first step. The present article describes indications whereby placental examination is warranted, mentions stepwise gross description of the placental cord,
\end{abstract}

Sunil Jaiman

suniljaiman@gmail.com

1 Department of Anatomic and Perinatal Pathology and Cytology, Fernandez Hospital, Unit 3, Plot 769, Road No. 44, Jubilee Hills, Hyderabad, Telangana State 500033, India membranes, and the disk and the usefulness of placental examination in perinatal death; a figurative narration and a template for placental grossing are included.

Keywords Gross examination of the Placenta . Indications of placenta examination - Stillbirth - Perinatal morbidity and mortality

\section{Introduction}

Pathological examination of the placenta is performed for fetal, maternal, and placental indications (Table 1). The purposes are to identify fetal or maternal disease, to provide prognosis for the current and future pregnancies, to evaluate the effect of maternal disease on the pregnancy, and for legal considerations [1]. Many placental lesions are diagnosed solely by gross examination, and the extent of the pathologic processes is best recognized on the whole specimen. Meticulous gross examination of a placenta prior to histologic sectioning enhances microscopic interpretation. Ideally, clinical information is available at the time of gross examination, including duration of gestation, fetal weight, and any problems related to the pregnancy, birth, or fetus/neonate [2].

\section{Storage of Placentas}

Some authorities recommend storage in plastic containers in a refrigerator at $4{ }^{\circ} \mathrm{C}$ [3] while others advocate immediate fixation in formalin [4]. Complete fixation of a whole placenta takes many days in adequate amounts of formalin. Primary formalin fixation makes placentas less infectious and easier to section, and the extent of infarction can be 
Table 1 Indications for histopathological examination of the placenta

\section{Maternal indications}

Systemic disorders with clinical concerns for mother and infant, e.g., gestational diabetes on insulin, pre-gestational diabetes, collagen disease, seizures, hypertensive disorders, severe anemia

Premature delivery $<34$ weeks of gestation

Peri-partum fever and/or infection

Suspicion of infection with HIV, syphilis, TORCH

Unexplained third trimester bleeding

Unexplained or recurrent pregnancy complications (fetal growth restriction, still birth, spontaneous miscarriage)

Abruptio placenta

Thick and/or viscid meconium

Severe oligohydramnios

Non elective pregnancy termination

Fetal/neonatal indications

Still birth or perinatal death

Hydrops fetalis

Birth weight $<10$ th centile

Major congenital anomalies, dysmorphic phenotype or abnormal karyotype

Twin growth discordancy $>20 \%$

Multiple gestation with same sex infants and fused placentas

Infection or sepsis

Seizures

Compromised clinical conditions (cord blood $\mathrm{pH}<7.0$; apgar score $<6$ at $5 \mathrm{~min}$; ventilator assistance for $>10$ min; severe anemia; hematocrit $<35 \%$ )

Placental indications

Physical abnormalities (shape abnormalities, infarct, mass, vascular thrombosis, retroplacental hematoma, amnion nodosum, abnormal coloration or opacification, malodor)

Small or large sized placenta

Umbilical cord lesions (thrombosis, torsion, true knot, single artery, absence of Wharton's jelly)

Cord length $<32 \mathrm{~cm}$ at term or abnormally long cord

assessed better. However, formalin fixation renders the placenta unsuitable for bacteriologic examination and tissue culture and has other disadvantages such as storage problems, expenses, and odor [2, 3].

\section{Grossing Technique}

Prior to grossing, a photographic record is desirable and useful. Photographs of placental abnormalities may be referred to later and correlated with microscopic features and clinical aspects. Hematoxylin and eosin (H\&E) stain is used for histological examination. Special assays such as chromosome analysis, mRNA and microarray analyses, and stereological analyses require special collection and sampling procedures, which are beyond the scope of this article.

Because placentas differ widely in shape, size, and in appearance, the neophyte must examine a large number of placentas to become familiar with the spectrum. There are various techniques to gross a placenta [1-3] and described below is the one followed by the author (Fig. 1). All placental specimens at the author's institute are fixed in $10 \%$ formalin. The placenta is checked for intactness, and the umbilical cord, membranes, and disk examined in succession. The reporting template used by the author is appended (Table 2).

\section{Cord}

The cord normally inserts into the placental disc in a central or near central location. Other variants include eccentric, marginal, velamentous, furcated, and interpositional cords. Premature infants tend to have thicker umbilical cords than more mature babies, whereas cord substance is often lacking and cords are thin in uteroplacental insufficiency. Edema of the cord can be significant in hydrops fetalis. Cord inflammation may manifest as a yellow discoloration or microabscesses when infected with Candida [2].

The length (including detached segments) and diameter (ranging from smallest to largest) of the cord are recorded. It must be remembered that the full cord is often not sent to the pathology laboratory. Long cords $(>75 \mathrm{~cm}$ at 

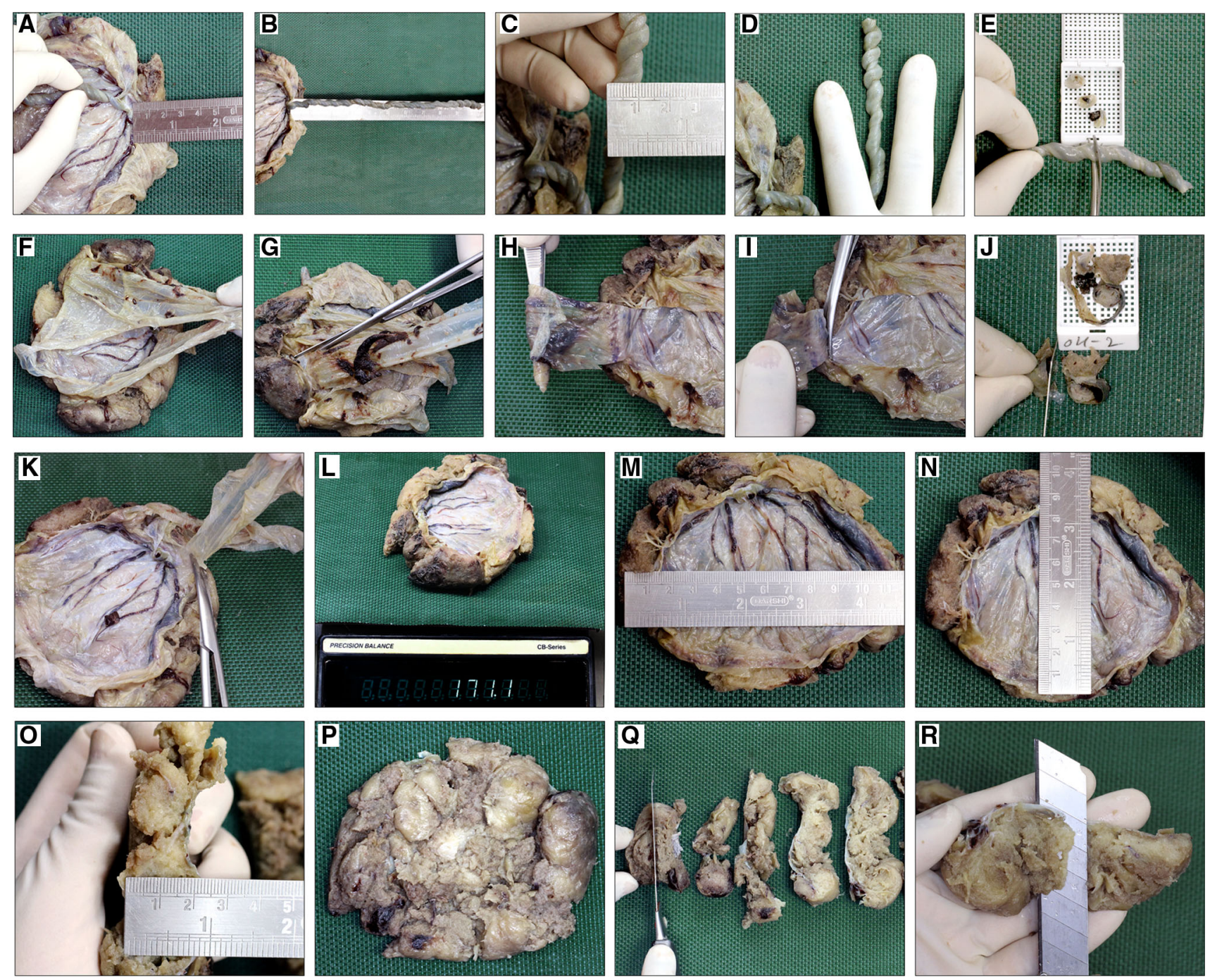

Fig. 1 Placenta grossing techniques (a-e cord, $\mathbf{f}-\mathbf{j}$ membranes, $\mathbf{k}-\mathbf{r}$ disk)

term) are usually associated with true knots and fetal entanglements. Congestion and thrombosis in cord vessels are helpful signs of true obstruction. Short cords are known to occur in disorders with decreased fetal movement and statistically show more problems with neurologic development [2]. Undue traction on a short cord can cause fetal distress, cord tearing with hemorrhage and, possibly, placental separation.

The spiraling of blood vessels is checked and the number of coils and direction of coiling recorded. Most commonly it is counterclockwise (left twist). Cords with significantly higher numbers of twists and, possibly, those without twists, have higher associated morbidity and mortality [5]. One must also examine for and record the presence of true knots, segmental narrowing/strictures, ulcerations, swellings, nodularity, and thrombi. Umbilical cord strictures are significant reductions in the size of the umbilical cord and are frequently found near the fetal end.
There is normally a gradual diminution of the Wharton's jelly towards the fetal abdomen but this assumes ominous clinical significance if associated with excessively long cords, hypercoiling, placental surface venous thrombi, and intimal fibrin cushions [6].

The cord is severed from the disk and sections taken from the cord insertion, $5 \mathrm{~cm}$ from the insertion and the fetal end. Any nodularity or abnormal-looking areas are also sampled. At this juncture, the number of vessels is noted. Sampling multiple areas is important because inflammatory response, thrombi, and other features are not always uniformly distributed throughout the length of the cord. Embryologic remnants are rarely visible grossly and most disappear leaving two umbilical arteries and the left umbilical vein. The absence of one umbilical artery is a common anomaly, occurring in approximately $1 \%$ of deliveries. It is more frequent with multiple gestation and velamentous cord insertions. Approximately $20 \%$ of 
Table 2 Template of grossing of the placenta

Received fresh / in formalin and labeled as placenta for HPE is an intact / fragmented singleton placenta with a normal, thin / thick, edematous, central / eccentric ( $\mathrm{cm}$ from the margin) / marginal / marginal velamentous / furcate / velamentous / interpositional cord ( $\mathrm{cm}$, diameter ranging from to $\mathrm{cm})$ with three/two vessels.

Received in the same container is / are detached segments of the cord (length of $\mathrm{cm}$ and diameter ranging from to $\mathrm{cm})$.

The cord has normal / decreased / increased coiling twists) towards the right/left; coiling index: $/ \mathrm{cm}$. There is normal presence / absence of periarterial Wharton's jelly. The cord displays (does not display) ulcer (s). There is / are (no) true knots / pseudoknots and the cord shows (does not show) segmental narrowing.

The membranes are intact / fragmented, thin / thick, delicate, glistening, translucent / opaque and supple. The rupture is $\mathrm{cm}$ from the free edge of the body of the placenta. The insertion is normal at the margins. There is (no) circummargination / circumvallation. There is (no) evidence of meconium staining / squamous metaplasia / amnion nodosum / amniotic polyps / amniotic bands.

The disk gm; fixed and trimmed; $\mathrm{X}$ $\mathrm{X}$ $\mathrm{cm})$ is roughly spherical / oval / reniform / triangular / square / bilobed / membranous (placenta membranacea). There is no / an accessory (succenturiate) lobe ( $\mathrm{X}$ $\mathrm{X}$ $\mathrm{cm})$.

The fetal surface is smooth, shiny and with / without prominent vessels. Some of the vessels appear thick firm cord-like (thrombosed). There is (no) evidence of subchorionic fibrin plaques, subchorial thrombosis or subamniotic hematoma. 
Table 2 continued

The maternal surface is intact, normally cotyledonous, dark red / grey white, rough / irregular /ragged or corrugated and firm. There is (no) evidence of basal plate fibrin deposition involving $\%$ of the maternal surface. There is (no) evidence of retroplacental hematoma located centrally / paracentrally / marginally.

Serial sections show normal spongy, brown / grey white, moist, homogeneous surface. There is (no) evidence of massive basal plate (classic) / transmural perivillous fibrin. There is (no) infarction ( $\mathrm{cm}$ in greatest dimension) involving $\%$ of parenchyma.

Cassette

Cassette

Cassette

Cassette

Cassette

Cassette

Cassette

Cassette

Cassette

Cassette 
infants missing one artery have other major congenital anomalies, which may involve any organ system. Several cuts along the cord length should confirm this finding because the two umbilical arteries frequently fuse just above the insertion site [2].

\section{Membranes}

The membranes are examined for intactness, insertion, color, thickness, and surface characteristics. The membranes normally insert at the peripheral margin of the villous tissue, which is usually the outer limit of the vascular plate. In circumvallation, there is a redundant, doubledback membrane fold with enclosed debris and old hemorrhage at the point of membrane insertion. In circummargination, there is a small ridge of fibrin where the membranes contact the extended placental surface.

Opacification of the normally-translucent membranes may be observed in the presence of chorioamnionitis. Meconium imparts a green color; however, in immature placentas, greenish or brownish discoloration is more often due to blood breakdown products (hematoidin and hemosiderin) following hemolysis [2, 3]. The membranes are visually inspected for squamous metaplasia, amnion nodosum, amniotic polyps, amniotic bands, and tumors. Other thickenings in the membranes may represent compressed fetuses and, rarely, retained intrauterine devices [2].

The point of rupture of the membranes is isolated, a ribbon cut from the edge to the margin of the disk and a roll prepared. A very short distance from the rupture point to the disk would indicate a lower-lying placenta, and vice versa $[1,3]$. The 'jelly roll' method to obtain a maximum amount of membranes with decidua capsularis was first described by Zeek and Assali [7]. At least two sections are submitted for processing in one cassette. The membranes are then trimmed from the disk.

\section{Disk}

The disk (fixed and trimmed) is weighed and measurements in three dimensions are recorded. Excessive amounts of maternal retroplacental clots must be removed before weighing. Placental weight can vary considerably because it is affected by fixation, the presence of cord, membranes, and loose clots, the amount of blood retained, and the intactness of the maternal surface. Placental weight tables are based upon trimmed weight. Fox and colleagues have shown that formalin fixation for $24 \mathrm{~h}$ increases the placental weight by $7.67 \%$; hence, fresh weight $=$ fixed weight $\times 0.929$ [8]. If the placenta is weighed in the fixed state, this should be noted in the report. The gestational age and weight of the baby are important to appropriately evaluate the measured weight, and the fetal/placental weight ratio can be readily calculated during the grossing process. The ratio increases from approximately 2 at 20 weeks of gestation to approximately $7-8$ at term $[9,10]$. A heavy or light placenta often indicates an abnormal pregnancy. Term placentas generally weigh between 400 and $600 \mathrm{~g}$.

The shape of the placenta is recorded at this juncture and accessory/succenturiate lobes looked for. Generally, the placenta is round to ovoid and approximately 18 - to $20-\mathrm{cm}$ diameter by $1.5-$ to $2.5-\mathrm{cm}$ thick at term [2]. The shape is assessed by measuring the length of the longest axis and then the longest length perpendicular to the first $[11,12]$. Ellipticity is related to developmental programming of the fetus and the shape of the placenta may reflect the site of implantation and hence, potentially, its maternal vascular supply [3, 13].

When studying the fetal surface of the placenta, one notes its color and looks for evidence of subchorionic fibrin plaques, subchorial thrombosis, and subamniotic hematomas. Ectatic thick-walled vessels of placental mesenchymal dysplasia may be apparent. The fetal surface of the mature placenta is often bosselated with tiny white elevations underneath the chorion, giving the surface a mosaic, irregular pattern. These protrusions represent accumulations of fibrin in the intervillous space, and they increase in number with advancing maturity. Large subchorionic thrombi are abnormal and occasionally associated with fetal growth restriction [3]. The fetal vessels carried in the chorion must be carefully inspected for thrombi which appear as white-yellow streaks on the surface of the vessel. Arteries are superficial to (cross over the surface of) veins. Cysts may be seen on the fetal surface and also within the parenchymal septae. These are caused by proliferation of the trophoblastic $\mathrm{X}$ cells and may, at times, become quite large and hemorrhagic [2, 3].

The intactness, color, and texture of the maternal surface are evaluated, and presence of retroplacental and marginal hematomas are ascertained. When bleeding is contained behind the placenta, the villous tissue becomes compressed by clot (distinguishing it from postpartum blood clot). The cotyledons increase in size and differentiation with advancing gestation. Any missing cotyledons should be noted. If no cotyledonary subdivisions exist in the mature placenta, it might indicate infiltration of the maternal floor with an excess amount of fibrin.

Multiple parallel sections are taken through the disk at $2-\mathrm{cm}$ intervals to evaluate the parenchyma. The color of villous tissue is almost wholly determined by its content of fetal blood. Thus, a congested placenta (as in maternal diabetes) is dark, while the placenta of an anemic, hydropic, exsanguinated, or erythroblastotic fetus is pale, much more friable and thicker $(3-5 \mathrm{~cm})$ in contrast to the normal placenta [3]. The cut surfaces are inspected for infarction, 
perivillous fibrin, and hemangiomas. Three-dimensional measurements of all lesions should be recorded, as well as, an estimation of the percentage of the placenta involved in a process such as infarction [1,3]. Most infarcts are usually based on the maternal surface and have rather linear defined margins. Infarcts feel firmer than the adjacent tissue and appear granular due to the remaining collapsed villi in varying stages of degeneration. Over time, the color changes from red to white. Cystic change and hemorrhagic regions may be seen in infarcts. Infarction is seen most commonly at the placental margin, where there is usually less blood flow. Intervillous thrombi occur in the intervillous space in central areas of the placenta. The earliest thrombi are fresh red clots, which progress through laminated thrombi to firm white lesions. Localized areas of perivillous fibrin deposition are seen in virtually all mature placentas and small amounts are not related to fetal or maternal disease. Massive perivillous fibrin deposition (MPVFD) typically results in small-forgestational age stiff placenta with firm yellow-white, thickened maternal surface. Three patterns of MPVFD are described: classic exhibiting basal fibrinoid along the entire maternal floor; borderline involving $25-50 \%$ of villi; transmural encasing $\geq 50 \%$ of villi and serial sections exhibit either basal fibrin or diffuse vertically oriented pale trabeculae extending from basal zone into middle and subchorial zones in a lattice-like network [14]. Avascular villi which imply interruption of the fetal blood supply manifest as pale areas but usually are not apparent grossly. Chorangiomas commonly occur under the chorionic plate. Hematogenous dissemination of fetal or maternal infection (e.g., listeria) may lead to abscesses and micro-abscesses scattered within the villous tissue.

Histologic samples of placental villous tissue should be from separate areas (different cotyledons) and not from the margin of the placenta, which frequently shows changes of diminished blood flow and fibrin deposition. The fetal surface of a villous section should include small blood vessels and be free of substantial subchorionic clot or fibrin. Sections are taken from the parenchyma in such a way that both chorionic and basal plates are included. If the placenta is too thick, the section is bisected and submitted in two consecutive cassettes. There is no definite consensus regarding the number of sections needed for adequacy [1$3,15]$. Placentas are heterogenous and overall evaluation can be significantly distorted by suboptimal sectioning. The very definition of chorangiosis warrants a minimum of three sections and focal pathology may be missed if not sectioned adequately, e.g., villitis of unknown etiology or borderline perivillous fibrin deposition may be missed if less than five sections are submitted. In addition to the unusual appearing areas, sections must be obtained from grossly normal portions of the placenta as well.

\section{Multifetal Gestation}

The placental combined weight should be compared with the ranges of combined placental weights for twin placentas (as matched for gestational week) [1]. The areas of the chorionic plate territories may be unequal, and this should be noted as relative percentages of the total placental surface. A recording of the membrane relation between the twins, triplets, and higher order multiples is mandatory. For meaningful analysis, it is necessary that the umbilical cords be labeled with sutures or clamps by the obstetrician, in the order of births.

The most important decisions to be made in examining the placentas of multiple births are: the number of membranes that divide the sacs (two or four) and the types of vascular anastomoses (generally present only in monochorionic twin placentas). If there are four distinctive leaves, it is a dichorionic diamniotic placenta, whereas if only two thin membranes are apposed, it is a monochorionic diamniotic placenta. In dichorionic diamniotic placentas, one usually finds a ridge at the site where the membranes meet over the placenta. This so-called ' $T$ zone' is best sampled by carefully incising the septum at about $3 \mathrm{~cm}$ above its attachment line and then cutting sections at right angles that include the chorionic plate tissue below, on both sides of the septum. Inclusion of the chorionic plate tissue, in the inverted ' $\mathrm{T}$ section', serves to stabilize it during sampling and to preserve its orientation during histologic processing. Submission of two such sections is recommended to ensure that a valid sample of the septum is available for microscopic interpretation [3].

The vascular 'equator' is the irregular interface of the limits of the branches of each twin's chorionic plate ramifications, and lies between the two cord insertion sites. The two halves of the twin placenta are dissected at the site of the vascular equator in order to determine the placental weight of each twin. The rest of the examination follows the standard protocol for the singleton placenta.

\section{Unexplained Intrauterine Fetal Demise (UIUFD)}

UIUFD is defined as fetal death occurring without any evidence of fetal, maternal, or placental pathology prior to the onset of labor and is classified as 'no relevant condition identified at birth' by the ReCoDe classification [16]. The placenta can provide exceedingly-useful information relating to perinatal morbidity and mortality. In a retrospective study of all $(n=211)$ stillbirths after 24 weeks of gestation between January 2011 and December 2012 at the study institute by the author (unpublished data), 33 cases of UIUFD were identified. The placenta was found to be the 
miscreant, directly contributing to demise in nearly $88 \%$ of the women presenting with UIUFD and the lesions identified were fetal blood supply abnormalities (42.4\%), maternal blood supply abnormalities (12.1\%), acute chorioamnionitis $(18.2 \%)$, and miscellaneous conditions such as massive perivillous fibrinoid, villitis of unknown etiology, etc. (15.2\%) [data presented at 60th PPS Annual Meeting (jointly with the SPP) at Birmingham UK in September 2014; 'Unexplained stillbirth-is placenta the miscreant?' which can be accessed at http://www.ppsspp2014.co.uk/wp-content/uploads/2014/02/ABSTRACTS4th-joint-PPS-SPP-Meeting-Bham-Sept-14.pdf)]. This clearly emphasizes the role of placental examination in evaluating the cause of stillbirths and reiterates the importance of methodical and meticulous examination of the placenta as detailed above.

Acknowledgments The author would like to thank the entire staff of the Department of Perinatal Pathology, Fernandez Hospital for their technical support; recognize V. Srinivas for taking gross pictures and creating a collage; and sincerely appreciate the assistance of Dr. G. Sirisha in administrative tasks.

\section{Conflict of interest None.}

\section{References}

1. Faye-Petersen OM, Heller DS, Joshi VV. Handbook of placental pathology. 2nd ed. London: Taylor and Francis; 2006.

2. Kaplan C. Gross examination of the placenta. Surg Pathol Clin. 2013;6:1-26.

3. Benirschke K, Burton GJ, Baergen RN. Examination of the placenta. In: Benirschke K, Kaufman P, editors. Pathology of the human placenta. 6th ed. Berlin: Springer; 2012. p. 1-12.

4. Bartholomew RA, Colvin ED, Grimes WH, Fish JS, Lester WM, Galloway WH. Criteria by which toxemia of pregnancy may be diagnosed from unlabeled formalin fixed placentas. Am J Obstet Gynecol. 1961;82:277-90.

5. Machin GA, Ackerman J, Gilbert-Barness E. Abnormal umbilical cord coiling is associated with adverse perinatal outcomes. Pediatr Dev Pathol. 2000;3(5):462-71.

6. Benirschke K, Burton GJ, Baergen RN. Anatomy and pathology of the umbilical cord. In: Benirschke K, Kaufman P, editors. Pathology of the human placenta. 6th ed. Berlin: Springer; 2012. p. $309-75$.

7. Zeek PM, Assali NS. Vascular changes in the decidua associated with eclamptogenic toxemia of pregnancy. Am J Clin Pathol. 1950;20:1099-109.

8. Fox GE, Van Wesep R, Resau JH, Sun CC. The effect of immersion formaldehyde fixation on human placental weight. Arch Pathol Lab Med. 1991;115:726-8.

9. Molteni RA, Stys SJ, Battaglia FC. Relationship of fetal and placental weight in human beings: fetal/placental weight ratios at various gestational ages and birth weight distributions. J Reprod Med. 1978;21(5):327-34.

10. Perry IJ, Beevers DG, Whincup PH, Bareford D. Predictors of ratio of placental weight to fetal weight in multiethnic community. BMJ. 1995;310(6977):436-9.

11. Pathak S, Hook E, Hackett G, Murdoch E, Sebire NJ, Jessop F, et al. Cord coiling, umbilical cord insertion and placental shape in an unselected cohort delivering at term: relationship with common obstetric outcomes. Placenta. 2010;31:963-8.

12. Salafia CM, Yampolsky M, Misra DP, Shlakhter O, Haas D, Eucker B, et al. Placental surface shape, function, and effects of maternal and fetal vascular pathology. Placenta. 2010;31:958-62.

13. Barker DJ, Thornburg KL, Osmond C, Kajantie E, Eriksson JG. The surface area of the placenta and hypertension in the offspring in later life. Int J Dev Biol. 2010;54:525-30.

14. Faye-Petersen OM, Ernst LM. Maternal floor infarction and massive perivillous fibrin deposition. Surg Pathol Clin. 2013;6:101-14.

15. Cox P, Evans C. Tissue pathway for histopathological examination of the placenta. UK: The Royal College of Pathologists; 2011.

16. Gardosi J, Kady SM, McGeown P, Francis A, Tonks A. Classification of stillbirth by relevant condition at death $(\mathrm{ReCoDe})$ : population based cohort study. BMJ. 2005;331:1113-7. 